\title{
MINIMAX DESIGN OF 2-D IIR DIGITAL FILTERS USING SEQUENTIAL SEMIDEFINITE PROGRAMMING
}

\author{
W.-S. Lu and A. Antoniou \\ Dept. of Electrical and Computer Engineering \\ University of Victoria \\ Victoria, BC, Canada V8W 3P6 \\ E-mail:wslu@ece.uvic.ca, aantoniou@shaw.ca
}

\begin{abstract}
A method for the minimax design of quadrantally symmetric 2-D IIR filters with guaranteed stability is proposed. The design problem is solved by formulating an objective function in the semidefinite programming framework using a linear approximation for the transfer function. The issue of filter stability is addressed by converting the stability constraints into linear matrix inequalities based on the wellknow Lyapunov stability theory.
\end{abstract}

\section{INTRODUCTION}

A great deal of research on the design of one- and twodimensional (1- and 2-D) digital filters has been carried out in the past [1]-[3]. The design of 2-D IIR filters presents two additional challenges over and above the design of 2-D FIR filters. First, while 2-D FIR filters are inherently stable, the stability of 2-D IIR filters is fairly difficult to formulate in a manner suitable for constrained optimization. Second, since the transfer functions of IIR filters are rational functions, the degree of nonlinearity involved in their design by optimization is considerably higher than that for FIR filters.

In this paper, we develop a method for the minimax design of IIR quadrantally symmetric 2-D filters with guaranteed stability. The method is essentially a sequential minimization algorithm and is as follows. At the $k$ th iteration a linear approximation for the transfer function is obtained using its Taylor expansion. In this way, an objective function can be deduced which is an upper bound of the approximation error in the frequency range of interest. The objective function is then minimized subject to several constraints. These constraints include a stability constraint and constraints on the filter's maximum passband ripple and minimum stopband attenuation. The stability constraint is converted into a linear matrix inequality (LMI) and it is shown that each iteration of the design can be performed using semidefinite programming (SDP). A design example is included to illustrate the proposed method.

\section{LINEAR APPROXIMATION FOR TRANSFER FUNCTION}

Consider a quadrantally symmetric 2-D IIR digital filter whose transfer function is given by

$$
H\left(z_{1}, z_{2}\right)=\frac{B\left(z_{1}, z_{2}\right)}{A\left(z_{1}\right) A\left(z_{2}\right)}
$$

where

$$
B\left(z_{1}, z_{2}\right)=\sum_{i=0}^{n} \sum_{k=0}^{n} b_{i k} z_{1}^{-i} z_{2}^{-k}
$$

and

$$
A(z)=\sum_{i=0}^{r} a_{i} z^{-i}, \quad a_{0}=1
$$

Because the filter is quadrantally symmetric, we have $b_{i k}=$ $b_{k i}$. Consequently, there are only $r+(n+1)(n+2) / 2$ variables in (1), which form a $[r+0.5(n+1)(n+2)]$ dimensional vector

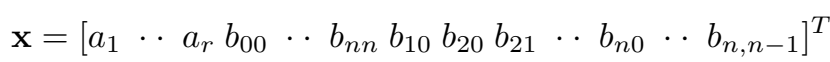

Denote vector $\mathbf{x}$ in the $k$ th iteration as $\mathbf{x}_{k}$ and the frequency response of the filter for $\mathbf{x}=\mathbf{x}_{k}$ as $H\left(e^{j \omega_{1}}, e^{j \omega_{2}}, \mathbf{x}_{k}\right)$. In the vicinity of $\mathbf{x}_{k}$, the design variable can be expressed as

$$
\mathbf{x}=\mathbf{x}_{k}+\boldsymbol{\delta}
$$

As in the approach used in [4] for the 1-D case, the frequency response can be approximated in terms of a linear function of $\boldsymbol{\delta}$ as

$$
H\left(e^{j \omega_{1}}, e^{j \omega_{2}}, \mathbf{x}\right) \approx H\left(e^{j \omega_{1}}, e^{j \omega_{2}}, \mathbf{x}_{k}\right)+\mathbf{g}_{k}^{T} \boldsymbol{\delta}
$$

where $\mathbf{g}_{k}$ is the gradient of $H\left(e^{j \omega_{1}}, e^{j \omega_{2}}, \mathbf{x}\right)$ for $\mathbf{x}=\mathbf{x}_{k}$, i.e.,

$$
\mathbf{g}_{k}=\nabla H\left(e^{j \omega_{1}}, e^{j \omega_{2}}, \mathbf{x}_{k}\right)
$$


From (1), the gradient vector can be evaluated as

$\frac{\partial H}{\partial x_{i}}=\left\{\begin{array}{l}-\frac{H}{A_{1}} e^{-j i \omega_{1}}-\frac{H}{A_{2}} e^{-j i \omega_{2}} \quad \text { for } 1 \leq i \leq r \\ \frac{1}{A_{1} A_{2}} e^{-j i\left(\omega_{1}+\omega_{2}\right)} \quad \text { for } r+1 \leq i \leq r+n+1 \\ \frac{1}{A_{1} A_{2}}\left[e^{-j\left(l \omega_{1}+m \omega_{2}\right)}+e^{-j\left(m \omega_{1}+l \omega_{2}\right)}\right] \quad \text { otherwise }\end{array}\right.$

where $H, A_{1}, A_{2}$ denote the values of $H\left(e^{j \omega_{1}}, e^{j \omega_{2}}\right)$, $A\left(e^{j \omega_{1}}\right)$, and $A\left(e^{j \omega_{2}}\right)$ evaluated at $\mathbf{x}=\mathbf{x}_{k}=\left[\begin{array}{llll}x_{1} & x_{2} & \cdots & x_{i}\end{array}\right.$ $\left.\cdots x_{n}\right]^{T}$, respectively.

\section{DESIGN ALGORITHM}

\subsection{Semidefinite Programming}

An SDP problem can be formulated in several ways. For the purpose of filter design, the formulation

$$
\begin{array}{ll}
\underset{\text { subject to }}{\operatorname{minimize}} & \mathbf{c}^{T} \mathbf{x} \\
\mathbf{F}(\mathbf{x})= & \mathbf{F}(\mathbf{x}) \succeq \mathbf{0} \\
& \mathbf{F}_{0}+\sum_{i=1}^{n} x_{i} \mathbf{F}_{i}
\end{array}
$$

where $\mathbf{c} \in R^{n \times 1}, \mathbf{F}_{i} \in R^{n \times n}$ for $0 \leq i \leq n$ are constant matrices with $\mathbf{F}_{i}$ symmetric, and $\mathbf{F}(\mathbf{x}) \succeq \mathbf{0}$ denotes that $\mathbf{F}(\mathbf{x})$ is positive semidefinite turns out to be convenient. Note that the constraint matrix is affine with respect to $\mathrm{x}$ and that SDP includes both linear and convex quadratic programming as special cases. More importantly, many interior-point methods that have proven efficient for linear programming have recently been extended to SDP [5]-[7]. Efficient and user-friendly software implementations of various SDP algorithms are available, for example, the LMI Control Toolbox [8], which works with MATLAB.

\subsection{Problem Formulation}

The minimax design can be obtained as the solution of the constrained optimization problem

$$
\underset{\mathbf{x}}{\operatorname{minimize}}\left[\operatorname{maximize}_{\left(\omega_{1}, \omega_{2}\right) \in \Omega} e\left(\omega_{1}, \omega_{2}, \mathbf{x}\right)\right]
$$

subect to: $H\left(e^{j \omega_{1}}, e^{j \omega_{2}}, \mathbf{x}\right)$ represents a stable filter

where

$$
\begin{aligned}
& e\left(\omega_{1}, \omega_{2}, \mathbf{x}\right)= \\
& W\left(\omega_{1}, \omega_{2}\right)\left|H\left(e^{j \omega_{1}}, e^{e \omega_{2}}, \mathbf{x}\right)-H_{d}\left(\omega_{1}, \omega_{2}\right)\right|^{2}
\end{aligned}
$$

$\Omega=\left\{\left(\omega_{1}, \omega_{2}\right):-\pi \leq \omega_{1}, \omega_{2} \leq \pi\right\}, H_{d}\left(\omega_{1}, \omega_{2}\right)$ is the desired frequency response, and $W\left(\omega_{1}, \omega_{2}\right) \geq 0$ is a weighting function. The problem in (3) can be reformulated as

$$
\begin{gathered}
\text { minimize } \quad \mu \\
\text { subject to: } \quad e\left(\omega_{1}, \omega_{2}, \mathbf{x}\right) \leq \mu \quad \text { for }\left(\omega_{1}, \omega_{2}\right) \in \Omega \\
H\left(e^{j \omega_{1}}, e^{j \omega_{2}}, \mathbf{x}\right) \text { represents a stable filter }
\end{gathered}
$$

where $\mu$ is treated as an additional design variable. By using the linear approximation for $H\left(e^{j \omega_{1}}, e^{j \omega_{2}}, \mathbf{x}\right)$ in (2), we can write

$$
e\left(\omega_{1}, \omega_{2}, \mathbf{x}\right) \approx \boldsymbol{\delta}^{T} \mathbf{Q}_{k} \boldsymbol{\delta}+2 \boldsymbol{\delta}^{T} \mathbf{q}_{k}+c_{k}
$$

where

$$
\begin{gathered}
\mathbf{Q}_{k}=W\left(\omega_{1}, \omega_{2}\right) \Re e\left(\overline{\mathbf{g}}_{k} \mathbf{g}_{k}^{T}\right) \\
\mathbf{q}_{k}= \\
W\left(\omega_{1}, \omega_{2}\right) \Re e\left\{\left[H\left(e^{j \omega_{1}}, e^{j \omega_{2}}, \mathbf{x}_{k}\right)-H_{d}\left(\omega_{1}, \omega_{2}\right)\right] \overline{\mathbf{g}}_{k}\right\} \\
c_{k}=e\left(\omega_{1}, \omega_{2}, \mathbf{x}_{k}\right)
\end{gathered}
$$

and the constraint in (4b) can be approximated as

$$
\left(\mu-2 \boldsymbol{\delta}^{T} \mathbf{q}_{k}-c_{k}\right)-\left(\mathbf{Q}_{k}^{\frac{1}{2}} \boldsymbol{\delta}\right)^{T}\left(\mathbf{Q}_{k}^{\frac{1}{2}} \boldsymbol{\delta}\right) \geq 0
$$

for $\left(\omega_{1}, \omega_{2}\right) \in \Omega$ where $\mathbf{Q}_{k}^{\frac{1}{2}}$ is the asymmetric square root of $\mathbf{Q}_{k}$. It can be readily shown that (6) holds if and only if

$$
\boldsymbol{\Phi}_{k}\left(\omega_{1}, \omega_{2}\right)=\left[\begin{array}{cc}
\mathbf{I} & \mathbf{Q}_{k}^{\frac{1}{2}} \boldsymbol{\delta} \\
\boldsymbol{\delta}^{T} \mathbf{Q}_{k}^{\frac{T}{2}} & \mu-2 \boldsymbol{\delta}^{T} \mathbf{q}_{k}-c_{k}
\end{array}\right] \succeq \mathbf{0}
$$

for $\left(\omega_{1}, \omega_{2}\right) \in \Omega$. If we denote the complex-valued gradient vector as $\mathbf{g}_{k}=\mathbf{g}_{k r}+j \mathbf{g}_{k i}$, then (5b) leads to

$$
\mathbf{Q}_{k}=W\left(\omega_{1}, \omega_{2}\right)\left(\mathbf{g}_{k r} \mathbf{g}_{k r}^{T}+\mathbf{g}_{k i} \mathbf{g}_{k i}^{T}\right)=\mathbf{G}_{k}^{T} \mathbf{G}_{k}
$$

where

$$
\mathbf{G}_{k}=W^{\frac{1}{2}}\left(\omega_{1}, \omega_{2}\right)\left[\begin{array}{ll}
\mathbf{g}_{k r} & \mathbf{g}_{k i}
\end{array}\right]^{T}
$$

and (7) becomes

$$
\boldsymbol{\Phi}_{k}\left(\omega_{1}, \omega_{2}\right)=\left[\begin{array}{cc}
\mathbf{I} & \mathbf{G}_{k} \boldsymbol{\delta} \\
\boldsymbol{\delta}^{T} \mathbf{G}_{k}^{T} & \mu-2 \boldsymbol{\delta}^{T} \mathbf{q}_{k}-c_{k}
\end{array}\right] \succeq \mathbf{0}
$$

for $\left(\omega_{1}, \omega_{2}\right) \in \Omega$. From (8) and (9) it is evident that $\boldsymbol{\Phi}_{k}\left(\omega_{1}, \omega_{2}\right)$ is a $3 \times 3$ symmetric matrix.

In the $k$ th iteration the optimization problem in (4) can be formulated as

$$
\operatorname{minimize} \hat{\mathbf{c}}^{T} \hat{\boldsymbol{\delta}}
$$

subject to: $\mathbf{S}_{k} \succeq \mathbf{0}$ 


$$
H\left(e^{j \omega_{1}}, e^{j \omega_{2}}, \mathbf{x}\right) \text { represents a stable filter }
$$

where

$$
\hat{\mathbf{c}}=\left[\begin{array}{c}
1 \\
0 \\
\vdots \\
0
\end{array}\right], \quad \hat{\boldsymbol{\delta}}=\left[\begin{array}{l}
\mu \\
\delta
\end{array}\right]
$$

and

$$
\mathbf{S}_{k}=\operatorname{diag}\left\{\boldsymbol{\Phi}_{k}\left(\omega_{1}^{(1)}, \omega_{2}^{(1)}\right), \ldots, \boldsymbol{\Phi}_{k}\left(\omega_{1}^{(L)}, \omega_{2}^{(M)}\right\}\right.
$$

with $\left\{\left(\omega_{1}^{(l)}, \omega_{2}^{(m)}\right)\right.$ for $\left.1 \leq l \leq L, 1 \leq m \leq M\right\}$ being a set of frequencies in the frequency region of interest.

\subsection{Stability Constraints}

The stability constraint in (10c) needs appropriate treatment to make it tractable for numerical optimization. Denote the vectors formed from the first $r$ components of $\mathbf{x}_{k}+\boldsymbol{\delta}$ by $\mathbf{a}_{k}+\boldsymbol{\delta}_{1}$. Since the denominator of $H\left(z_{1}, z_{2}\right)$ is separable, it can be shown [9] that the IIR filter with coefficient vector $\mathbf{x}_{k}+\boldsymbol{\delta}$ is stable if and only if the magnitudes of the eigenvalues of matrix

$$
\mathbf{D}_{k}=\left[\begin{array}{c}
-\left(\mathbf{a}_{k}+\boldsymbol{\delta}_{1}\right)^{T} \\
\hat{\mathbf{I}}_{r}
\end{array}\right]
$$

are all strictly less than one, where $\hat{\mathbf{I}}_{r}$ denotes a matrix of size $r \times(r+1)$ obtained by augmenting the identity matrix with a zero column on the right. In such a case, $\mathbf{D}_{k}$ is said to be a stable matrix. Applying the well-known Lyapunov theory [9], one concludes that matrix $\mathbf{D}_{k}$ is stable if and only if there exists a positive definite matrix $\mathbf{P}$ such that

$$
\mathbf{P}-\mathbf{D}_{k}^{T} \mathbf{P} \mathbf{D}_{k} \succ \mathbf{0}
$$

where $\mathbf{M} \succ \mathbf{0}$ denotes that matrix $\mathbf{M}$ is positive definite. It can be readily verified that the matrix inequality in (11) holds if and only if

$$
\left[\begin{array}{cc}
\mathbf{P}^{-1} & \mathbf{D}_{k} \\
\mathbf{D}_{k}^{T} & \mathbf{P}
\end{array}\right] \succ \mathbf{0}
$$

To assure a stability margin for the IIR filter, the constraints in (12) are modified as

$$
\mathbf{Y}_{k}=\left[\begin{array}{cc}
\mathbf{P}^{-1}-\tau \mathbf{I}_{r} & \mathbf{D}_{k} \\
\mathbf{D}_{k}^{T} & \mathbf{P}-\tau \mathbf{I}_{r}
\end{array}\right] \succeq \mathbf{0}
$$

where $\tau$ is a positive scalar that controls the stability margin of the filter.

The problem in (10) can now be converted to

$$
\begin{aligned}
& \text { minimize } \hat{\mathbf{c}}^{T} \hat{\boldsymbol{\delta}} \\
& \text { subject to: } \quad\left[\begin{array}{cc}
\mathbf{S}_{k} & \mathbf{0} \\
\mathbf{0} & \mathbf{Y}_{k}
\end{array}\right] \succeq \mathbf{0}
\end{aligned}
$$

From (7), (10), and (13), we see that matrices $\mathbf{S}_{k}$ and $\mathbf{Y}_{k}$ depend on vector $\hat{\boldsymbol{\delta}}$ affinely. The matrix $\mathbf{P}$ in (13) is not considered as design variable. Rather, this positive definite matrix is fixed in each iteration and can be obtained, for example, by solving the Lyapunov equations

$$
\mathbf{P}-\hat{\mathbf{D}}_{k}^{T} \mathbf{P} \hat{\mathbf{D}}_{k}=\mathbf{I}
$$

where

$$
\hat{\mathbf{D}}_{k}=\left[\begin{array}{c}
-\mathbf{a}_{k}^{T} \\
\hat{\mathbf{I}}_{r_{1}}
\end{array}\right]
$$

It is well-known [9] that if the IIR filter with coefficient vector $\mathbf{x}_{k}$ is stable, then the solution of the equation in (15) is unique and positive definite.

With $\mathbf{P}$ fixed in $\mathbf{Y}_{k}$, the minimization problem in (15) is an SDP problem of size $1+r+0.5(n+1)(n+2)$.

\subsection{Design Steps}

Given the order of the IIR filter $(n, r)$ and the desired frequency response $H_{d}\left(\omega_{1}, \omega_{2}\right)$, the proposed design method starts with an initial point $\mathbf{x}_{0}$ that corresponds to a stable design obtained using a conventional method. For example, one can design an FIR filter of order $n$ to approximate $H_{d}\left(\omega_{1}, \omega_{2}\right)$ and simply set $A\left(z_{1}\right) \equiv 1$ and $A\left(z_{2}\right) \equiv 1$ as the initial design. With this $\mathbf{x}_{0}$, a positive definite matrix $\mathbf{P}$ can be obtained by solving the Lyapunov equation in (15), and quantities $\mathbf{G}_{k}, \mathbf{q}_{k}$, and $c_{k}$ can be evaluated by using (5) and (8). Next the SDP problem in (14) is solved. The solution obtained $\hat{\boldsymbol{\delta}}^{*}=\left[\begin{array}{ll}\mu^{*} & \boldsymbol{\delta}^{* T}\end{array}\right]^{T}$ can be used to update $\mathbf{x}_{0}$ to $\mathbf{x}_{1}=\mathbf{x}_{0}+\boldsymbol{\delta}^{*}$. The iteration continues until $\left\|\boldsymbol{\delta}^{*}\right\|$ is less than a prescribed tolerance $\varepsilon$.

\section{A DESIGN EXAMPLE}

The proposed method was applied to design several types of 2-D IIR filters, including circularly symmetric lowpass, highpass, and bandpass filters, and diamond-shaped lowpass filters. The design example presented here is a diamondshaped lowpass filter of order $(n, r)=(20,16)$ with normalized passband and stopband edges $\omega_{p}=0.8 \pi$ and $\omega_{a}=\pi$, respectively. The desired frequency response was assumed to be

$H_{d}\left(\omega_{1}, \omega_{2}\right)= \begin{cases}e^{-j 12\left(\omega_{1}+\omega_{2}\right)} & \text { for }\left(\omega_{1}, \omega_{2}\right) \text { in passband } \\ 0 & \text { for }\left(\omega_{1}, \omega_{2}\right) \text { in stopband }\end{cases}$

The weighting function used was

$W\left(\omega_{1}, \omega_{2}\right)= \begin{cases}1 & \text { for }\left(\omega_{1}, \omega_{2}\right) \text { in passband and stopband } \\ 0 & \text { for }\left(\omega_{1}, \omega_{2}\right) \text { elsewhere }\end{cases}$

The initial design was obtained by designing a 2-D FIR filter of order $(20,20)$ using the singular-value decomposition method [10] and setting $A_{1}\left(z_{1}\right) \equiv 1$ and $A_{2}\left(z_{2}\right) \equiv 1$. 
To implement the proposed algorithm, a total of 1098 frequency points that were uniformly distributed in the passband and stopband were used in the LMI constraints $\mathbf{S}_{k} \succeq$ 0. The SDP involved in the design was implemented using MATLAB LMI Control Toolbox [8]. With $\varepsilon=0.75 \times 10^{-4}$, it took the algorithm 55 iterations to converge to a solution. The amplitude response and the passband group-delay characteristic are depicted in Fig. 1. From the figure, it is observed that an approximately equirriple frequency response with an approximately constant passband group delay have been achieved. The passband ripple was found to be 0.0312 $\mathrm{dB}$ and the stopband attenuation was $36.05 \mathrm{~dB}$. The maximum pole magnitude was 0.8661 .

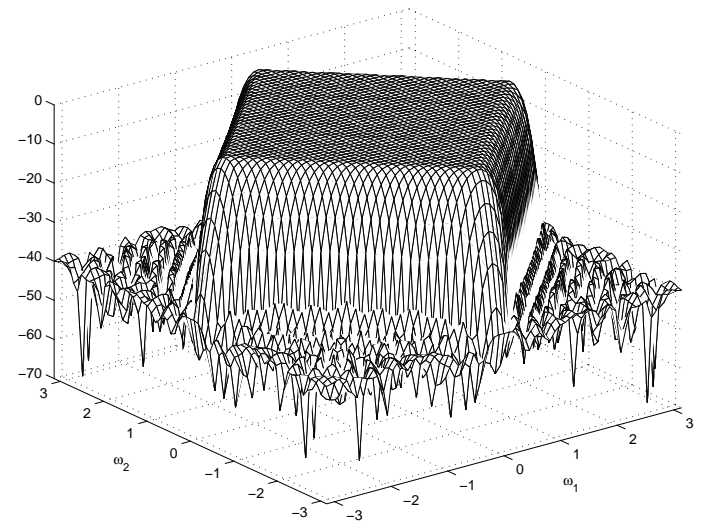

(a)

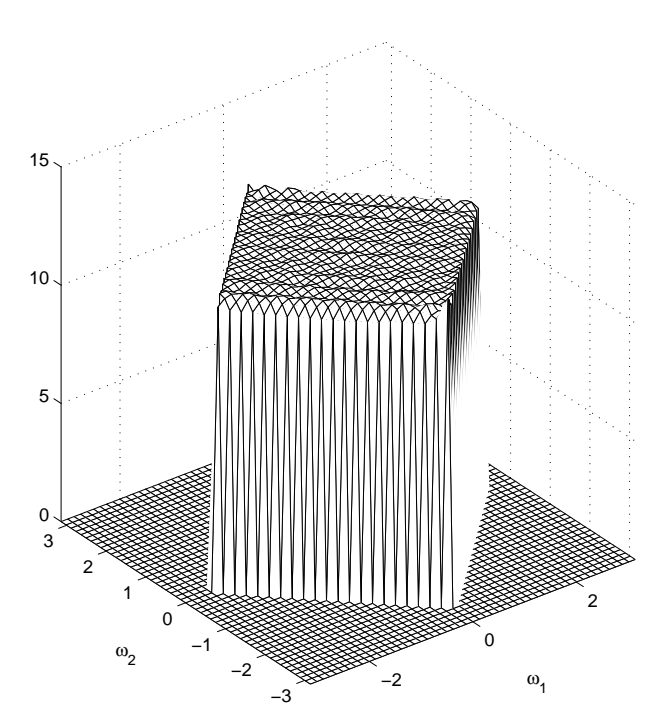

(b)

\section{CONCLUSION}

A method for the minimax design of IIR 2-D digital filters has been presented. The method is based on a sequential application of minimizing a linear approximation of the objective function using SDP. It has been demonstrated that the algorithm converges quickly to a solution and yields a filter with satisfactory performance and guaranteed stability.

\section{Acknowledgment}

The authors would like to thank the Natural Sciences and Engineering Research Council of Canada for supporting this research.

\section{REFERENCES}

[1] A. Antoniou, Digital Filters: Analysis, Design, and Applications, 2nd ed., Mc-Graw-Hill, 1993.

[2] A. V. Oppenheim and R. W. Schafer, Discrete-Time Signal Processing, Prentice-Hall, 1989.

[3] W.-S. Lu and A. Antoniou, Two-Dimensional Digital Filters, Marcel Dekker, 1992.

[4] M. Lang, "Algorithms for the constrained design of digital filters with arbitrary magnitude and phase responses", Doctoral dissertation, Vienna Univ. of Technology, June 1999.

[5] L. Vandenberghe and S. Boyd, "Semidefinite programming”, SIAM Review, vol. 38, pp. 49-95, March 1996.

[6] H. Wolkowicz, R. Saigal, and L. Vandenberghe (ed.), Handbook on Semidefinite Programming, Kluwer Academic, 2000.

[7] A. Ben-Tal and A. Nemirovski, Lectures on Modern Convex Optimization, SIAM, 2001.

[8] P. Gahinet, A. Nemirovski, A. J. Laub, and M. Chilali, Manual of LMI Control Toolbox, The MathWorks Inc., Natick, MA, 1995.

[9] T. Kailath, Linear Systems, Prentice-Hall, 1981.

[10] W.-S. Lu and A. Antoniou, "Application of the singular value decomposition in the design of two-dimensional digital filters", in Control and Dynamic Systems, ed. by C. T. Leondes, vol. 69, pp. 181-210, Academic Press, 1995.

Fig. 1. (a) Amplitude response and (b) passband groupdelay characteristic. 